\title{
Por el dolor que nos representa a todos: el movimiento por los derechos de género en una ciudad del noroeste de México
}

For the pain that represents us all: the movement for gender rights in a city in northwestern Mexico

\author{
Sheila Delhumeau Rivera ${ }^{1}$, Nelly Calderón de la Barca Guerrero² y \\ Mónica Lacavex Berumen ${ }^{3}$
}

\begin{abstract}
Resumen
En 2015 se registró un incremento de crímenes violentos contra las mujeres en Baja California, ante lo cual organizaciones y activistas sociales marcharon para visibilizar la violencia de género en varias ciudades del estado. Desde entonces, se articuló un movimiento que sigue trabajando, buscando influir en la conciencia ciudadana y en las políticas públicas.

Mediante la teoría de los movimientos sociales analizamos el proceso de construcción de la identidad y agenda de acción colectiva en torno a los derechos de género en Ensenada, con una metodología cualitativa a partir de entrevistas con actores sociales claves, la observación participante y la revisión de fuentes secundarias.
\end{abstract}

Palabras clave: movimientos sociales, activismo, proyecto político, derechos de género, violencia de género.

\footnotetext{
1 Doctora en Ciencias Sociales, labora actualmente como profesora-investigadora de tiempo completo en la Universidad Autónoma de Baja California y es líder del CA Sociedad y Gobierno. Se le puede contactar en la siguiente dirección: Facultad de Ciencias Administrativas y Sociales, Blvd. Zertuche y Blvd. de los Lagos s/n, Fracc. Valle Dorado, Ensenada, México, C.P. 22890; o por correo electrónico: sheila@uabc.edu.mx

2 Doctora en Ciencias Humanas Especialidad en Estudio de las Tradiciones, labora actualmente como profesora-investigadora de tiempo completo en la Universidad Autónoma de Baja California y es coordinadora de la Licenciatura en Comunicación. Se le puede contactar en la siguiente dirección: Facultad de Ciencias Administrativas y Sociales, Blvd. Zertuche y Blvd. de los Lagos s/n, Fracc. Valle Dorado, Ensenada, México, C.P. 22890; o por correo electrónico: nellycalderon@uabc.edu.mx

3 es Doctora en Estudios del Desarrollo Global, se desempeña actualmente como Vicerrectora del Campus Ensenada de la Universidad Autónoma de Baja California. Se le puede contactar en la siguiente dirección: Carretera Transpeninsular Ensenada - Tijuana No. 3917, Colonia Playitas, C.P. 22860; o por correo electrónico:monical@uabc.edu.mx
} 


\begin{abstract}
In 2015 there was an increase in violent crimes against women in Baja California and some organizations and social activists marched to make visible gender violence in several cities of the state. Since then, a movement has been articulated, seeking to influence citizen awareness and public policies.
\end{abstract}

Through the theory of social movements we analyze the process of construction of identity and collective action agenda around gender rights in Ensenada, with a qualitative methodology based on interviews with key social actors, participant observation and the review from secondary sources.

Keywords: social movements, activism, political project, gender rights, gender violence.

\title{
Introducción
}

A varias décadas de la reinstalación de los gobiernos democráticos en América Latina, el debate sobre los procesos de participación política de los distintos actores sociales adquiere relevancia. La consolidación del modelo neoliberal en la tarea de gobernar de estos países, así como el viraje hacia la derecha en algunas sociedades, nos llevan a voltear la mirada a los nuevos (o rejuvenecidos) actores y movimientos sociales que luchan por espacios mayores de equidad. Movimientos por los derechos de los indígenas, en la defensa del medioambiente, de la justicia y respeto a los derechos humanos, así como los movimientos sectoriales de mujeres y de jóvenes, son algunas manifestaciones contemporáneas de estos actores sociales, en contextos de autoritarismo, centralización de decisiones y crisis de representación en la democracia latinoamericana.

La teoría existente sobre movimientos sociales de principios del siglo pasado no era suficiente para explicar las nuevas condiciones, por lo que dio paso a enfoques emergentes entre los que se cuenta la teoría de los nuevos movimientos sociales (NMS). Este enfoque trasciende los fenómenos de movimientos sociales como manifestaciones del movimiento obrero y pone atención a las motivaciones de los actores sociales para organizarse en un sistema donde la diversificación del conflicto es evidente.

Desde marzo de 2015, se registró un incremento de crímenes 
pISSN: 2395-8758

eISSN: en trámite

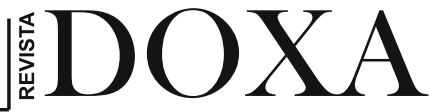

violentos contra las mujeres en Baja California, México, ante lo cual diversas organizaciones y activistas sociales exigieron que se tomaran medidas de emergencia. En el verano del mismo año, ante la falta de soluciones gubernamentales, se realizó en varias ciudades del estado una marcha demandando atención a la problemática de los derechos de las mujeres, a partir de la cual se ha articulado un movimiento que ha seguido trabajando, buscando influir en la conciencia ciudadana y en las políticas públicas.

En esta coyuntura social y a partir del fenómeno social que representó la marcha, se produjo en la comunidad la articulación con demandas complementarias sobre la equidad de género de otros colectivos, quienes encontraron en este movimiento el espacio para visibilizar sus acciones vinculadas al reconocimiento y los derechos de la diversidad sexual. Esto conllevó al surgimiento de formas de acción colectiva que fortalecieron la presencia y el discurso público de los activistas, transformando la discusión pública sobre la cuestión de género, la equidad y los derechos humanos en la región.

El presente artículo es parte de una investigación más amplia titulada "Liderazgos comunitarios, actores y experiencias en los municipios de Ensenada, Ciudad Juárez y Nuevo Casas Grandes" de la Red PRODEP Estudios Transfronterizos del Norte de México, compuesta por Cuerpos Académicos de la Universidad Autónoma de Baja California, Universidad Autónoma de Chihuahua, Universidad Autónoma de Ciudad Juárez y Universidad Autónoma de Sinaloa. En este documento se presentan los resultados del estudio en Ensenada.

\section{Movilización social y activismo por los derechos de género en México}

Varios estudios apuntan a la importancia de las acciones de grupos sociales organizados y actores sociales formales e informales en la formulación de políticas públicas y la reivindicación de derechos de ciudadanía en el estado de Baja California (Delhumeau, 2012; Moreno, 2008; Reyes, 2006). Dalton menciona que las dimensiones tradicionales de la participación política se identifican como: 1) el deber ciudadano (normas de orden social, de las cuales el votar está fuertemente relacionado), y 2) el compromiso ciudadano que abarca medidas de participación activa por grupos de la sociedad civil o de política con un grado de solidaridad, en la actualidad enfrentan "nuevas 


\section{DOXA}

pISSN: 2395-8758

eISSN: en trámite

presiones a niveles locales, nacionales e internacionales, asimismo parámetros de acción que posibilitan y hacen necesarios nuevos escenarios para el ejercicio de la ciudadanía" $(2008,1)$.

Adicionalmente, menciona que, en la práctica, la ciudadanía tradicional ha llevado a la exclusión o marginalización de grupos minoritarios y vulnerables, tales como mujeres, jóvenes e indígenas. En el caso particular de las mujeres mexicanas, Hernández (2006) señala que la idiosincrasia y la cultura son dos de los factores sociológicos que impiden la verdadera inclusión de las mujeres en los procesos de ciudadanización, refiriéndose a los factores enraizados en el sistema heteropatriarcal vinculado (y al servicio) del mismo sistema económico.

"La globalización también facilita, aumenta la visibilidad política de la afirmación cultural y de los derechos a la diferencia... [que] pueden coexistir con el reclamo de nuevos derechos sociales" (Aguirre, 2003, 13-14).

Las mujeres, al igual que los jóvenes y personas indígenas, pueden ser más capaces de ejercer una ciudadanía activa, aunque sus reclamos de bienestar y autonomía enfrentan dificultades. Inclusive, Aguirre considera el acceso al capital simbólico como un nuevo campo para el ejercicio de la ciudadanía y, por ello, concluye que el gran desafío actual es "unir la redistribución de los recursos materiales y la distribución de bienes simbólicos --en especial la participación" $(2003,14)$.

Las acciones de participación (sobre todo aquellas alternas a los mecanismos estatales) y el desarrollo de una conciencia (de clase, de género, social, cultural) y pertenencia, contribuyen a la ciudadanización. El carácter activo de la ciudadanía es indicador de procesos de cambio social en potencia, que puedan transformarse eventualmente "en la participación real y efectiva y, en consecuencia, en la acción política" (Oraisón, 2011, 84).

\section{Teoría de los nuevos movimientos sociales}

El análisis de los nuevos movimientos sociales, desprendido del enfoque moderno de los movimientos sociales del siglo XIX y primera mitad del siglo $\mathrm{XX}$, acentúa "los aspectos de ideario y proyecto de los movimientos sociales, vistos como actores y diferenciados de los 'antiguos' movimientos sociales a partir del tipo de activistas que engloban y las líneas de conflicto social en que se desenvuelven" (Delhumeau, 2012,57). Los nuevos movimientos 
sociales son formas de acción colectiva que emergieron en los años sesenta del siglo pasado, incorporando demandas que trascienden las necesidades de bienes materiales y el trabajo, moviéndose en áreas de reivindicación de derechos e ideologías.

Charles Tilly (1995) comenta que los nuevos movimientos sociales surgen en coyunturas de tensión social y de crisis. Entre los teóricos de los nuevos movimientos sociales se pueden encontrar una diversidad de posiciones: las teorías europeas de la identidad, con autores como Touraine (1981), Melucci (1985) y Offe (1985); o la teoría de la movilización de recursos americana, representada por McCarthy y Zald (1977) y Tarrow (1998), entre otras y con sus diferencias al interior de las mismas.

Para Touraine (1981) el actor social colectivo se define por su participación en un conflicto de relevancia histórica, tendiente a transformar el espacio físico y social del entorno. En el estudio de los nuevos movimientos sociales se acentúa el entendimiento de sus idearios y proyectos dentro del marco estructural que provee oportunidades y restricciones para la acción, así como a partir del cual construyen sus redes de apologistas y antagonistas. Para estos autores de la identidad lo fundamental es la búsqueda del conflicto en la acción colectiva, ya que en este y en las interacciones se condensan los ordenadores de la vida social.

Melucci (1985) al respecto propone la construcción de los sistemas de acción de los movimientos para comprender la naturaleza de la acción colectiva, para lo cual desarrolla el concepto de acción en función de las aspiraciones, de los recursos y obstáculos que enfrentan los actores situados en un sistema de oportunidades y restricciones, de tal manera que la organización se vuelve el punto crítico de observación para encontrar las relaciones fundamentales entre las acciones individuales que dan vida a la acción social.

Para Melucci (1988), los actores sociales de estos movimientos con metafóricamente nómadas, ya que sus movilizaciones son coyunturales, presentes y, por lo tanto, muchas veces temporales y reemplazables.

De esta manera, el comportamiento colectivo debe comprenderse como el resultado de un sistema de relaciones, orientaciones y significados diversos; analizarse el proceso desde la conformación del actor colectivo, implica explicar el movimiento o acción a partir de una pluralidad de aspectos, sus 


\section{DOXA}

pISSN: 2395-8758

eISSN: en trámite

combinaciones y su continuidad para redescubrir el papel creativo del actor en la sociedad contemporánea.

Los movimientos por los derechos de género surgen en la segunda mitad del siglo XX en los países europeos y en Estados Unidos, como una respuesta a la ideología patriarcal intrínseca a la vida cotidiana. Buscan reivindicar y hacer valer los derechos humanos fundamentales, donde se estipula la igualdad entre mujeres y hombres, por lo que hacen énfasis en la necesidad de promover políticas que garanticen la equidad en el acceso al trabajo, el mercado y la erradicación de la violencia.

Estos movimientos enfrentan resistencias sociales al buscar una transformación ideológica radical en la forma como se concibe el rol social de los géneros. Otra de las limitaciones que enfrentan es el significado polisémico del término en la vida diaria.

Entendemos el género como una serie de atributos y funciones, que van más allá de lo biológico/reproductivo, construido social y culturalmente; que es adjudicado a los sexos para justificar diferencias y relaciones de opresión entre los mismos.

El género se interioriza a través de la socialización: en un complejo y detallado proceso cultural de incorporación de formas de representarse, valorar y actuar en el mundo. Corresponde a un proceso que ocurre a lo largo del ciclo de vida, por lo que las relaciones de género son cambiantes en el tiempo.

De Keijzer (1998) menciona que existen un sinnúmero de transiciones que afectan las relaciones de género, como pueden ser: los procesos de urbanización, la incorporación de las mujeres a la fuerza laboral, el deterioro del poder adquisitivo, la creciente migración interna y a otros países, los cambios en la organización familiar, las tendencias a relaciones de género de mayor equidad en campos cada vez más diversificados, como la educación y la política.

Silvia López (2002) ha realizado en varios momentos un análisis del movimiento feminista en Baja California, el cual data de la mitad de la década de los años setenta, y adquiere fuerza y se consolida en los ochenta a través del movimiento urbano popular. A partir de la década de 1990 se da un proceso de "onegeización" (López, 2002, 210) donde se especializan y profesionalizan, buscando influir en las políticas públicas. López señala 
pISSN: 2395-8758

eISSN: en trámite

factores como la migración, la industria maquiladora y la consecuente demanda de mano de obra femenina, y la proliferación de asentamientos irregulares como elementos que impulsaron el movimiento feminista, que incluía a todas aquellas organizaciones de mujeres que compartían la idea de que el género era un asunto de interés político y social.

La novedad del movimiento feminista en el año 2000 en Baja California, son los procesos de incorporación de nuevos grupos, actores y demandas específicas relacionadas con condiciones sociales de desigualdad, falta de equidad y violencia, así como el relevo generacional, visibilidad en la calle y a través de redes sociales.

\section{Liderazgo y activismo social}

Para Gallo, Correa \& Pacheco (2015) la comunidad la constituye un grupo, creador de su propio espacio sociocultural a partir del establecimiento de sus propias reglas para relacionarse; de tal manera que asumen colectivamente el control y beneficio de sus recursos para la reproducción social.

Al hablar de liderazgo, es hacer referencia a la capacidad de las personas de lograr afianzar la identidad de grupo y llevar a cabo acciones. El liderazgo implica una serie de roles que desempeñados por una o varias personas, contribuyen al éxito grupal (Molina, Samper, \& Mayoral, 2013).

Como parte de los procesos de empoderamiento de las mujeres, los liderazgos femeninos son cada vez más visibles y más influyentes en la sociedad, si bien hay distintos tipos de liderazgos, como el político, social, empresarial; el liderazgo social o comunitario de las mujeres ha contribuido en gran medida al establecimiento y desarrollo de las comunidades, que han mostrado la presencia femenina como líderes sociales, así como gestoras y cuidadoras de la misma (Pedrín, Delhumeau, Spears, \& Martinez, 2014).

Lamas (2004) señala la importancia de contar con liderazgos de mujeres que de la mano con grupos vulnerados como jóvenes, indígenas, tercera edad, entre otros, reflexionen y concienticen, para que se convenzan que puede ser buenas líderes y sean capaces de convencer, alentar, abrir perspectivas, clarificar ideas, atraer seguidores y detectar nuevos grupos para impulsarlos.

De acuerdo a Becerra y Mata (2014) el liderazgo social no se 


\section{DOXA}

pISSN: $2395-8758$

eISSN: en trámite

impone por designación de una persona, sino que es la resultante de combinar sus atributos o cualidades en el quehacer cotidiano, con sus valores sociales. De esta manera el ser carismática, aceptada y conductora, no es una cuestión de suerte: es el resultado de actuar democráticamente, de ser transparente, constante y solidaria, de ser promotora de la participación ciudadana en la toma de decisiones estratégicas; de ser ética y equitativa, de tener un enfoque o visión de derechos humanos y de ser creyente y practicante de la perspectiva de género.

De acuerdo a la perspectiva de las autoras, es precisamente este tipo de liderazgo que se forma de mujeres con confianza en ellas mismas, o a su vez forma mujeres más seguras y autónomas, que influye la subjetividad femenina anteponiéndose a su destino supuestamente natural de ser dependientes y un ser para otros, que las hacen dudar sobre su independencia, que las destinaba a actividades de servicio y no de liderazgo.

Para Freire (2008, citado en Rojas, 2013) los líderes comunitarios surgen: a) en momentos de crisis de la comunidad, referidas tanto a crisis macrosociales como mesosociales que denotan necesidades y problemas críticos en la comunidad, y b) como producto del propio proceso de desarrollo comunitario que fortalece a los miembros y facilita la emergencia de nuevos líderes. En este sentido, el liderazgo crece a nivel del crecimiento del todo o es sustituido por nuevos líderes que emergen, en base a una nueva percepción social que van construyendo conjuntamente.

En lo que concierne a las características de los líderes comunitarios, Rojas Andrade (2013) observó que las personas que ejercen este rol emprenden acciones en torno al cumplimiento de las metas de la comunidad a través del ejercicio de prácticas transformacionales destinadas a la movilización y al aumento de la participación social, motivadas por fuertes convicciones y valores sociales cediendo su protagonismo individual a favor del de la comunidad misma.

El liderazgo comunitario (LC) es un proceso que opera al menos en dos niveles del agregado social, situación similar al concepto de empoderamiento (Silva \& Loreto, 2004). Desde el nivel individual, el LC se entiende como un liderazgo por la comunidad encarnado por los líderes que trabajan con y para otros, con el afán de alcanzar el cambio social. Estos son los líderes visibles, los que reciben la legitimación y los que simbolizan a la 
pISSN: 2395-8758

eISSN: en trámite

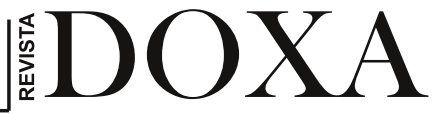

comunidad otorgándole un cuerpo y una voz cuando más lo necesita, es decir, en momentos de crisis y cuando el capital social acumulado requiere ser invertido en la realización de alguna meta (Andrade, 2013).

\section{Proyectos politicos de las organizaciones}

De acuerdo a Bolos (2003) las acciones colectivas son producto de interacciones complejas tanto al interior como al exterior de las organizaciones. Para estudiar las acciones colectivas organizadas, "resulta central la forma en que los actores las orientan" (p.57). Es por esto que es fundamental comprender los elementos estructurales y los recursos simbólicos en los cuales construyen su acción, ya que estos posibilitan o limitan las maneras como los colectivos se accionan y enfrentan a las mismas estructuras. "Un elemento importante que forma parte de los proyectos, son las elaboraciones ideológicas que los propios actores producen" (Bolos, 2003, 58).

Como otros estudiosos de los movimientos sociales señalan, el proceso de formación de las acciones colectivas inicia con la identificación personal de un problema social que se transforma en demanda, ante una situación de escasez de recursos materiales o simbólicos. "Entre los que forman el grupo inicial, el reconocimiento se produce sobre la base de una identificación con ellos mismos y de un modo de entender y de realizar el mundo: hay, pues, una forma similar -aunque no idéntica de analizar la realidad y las experiencias vividas en el pasado" (Bolos, 2003, 59). Este reconocimiento y la posterior construcción de una identidad colectiva, sin embargo, no se construye únicamente de similitudes como la clase, las condiciones de vida o las actividades que desarrollan, sino que se genera al compartir una definición de sí mismos y del sistema externo como adversario: la dimensión antagonista del movimiento que Melucci ubicaba "a nivel de lo simbólico" (Bolos, 2003).

Aun cuando las metas pueden ser o no ser alcanzada por los movimientos sociales, de acuerdo a Alberoni "la concreción de un movimiento en una organización permite hacer realidad su propio proyecto; a este deberemos buscarlo en el centro del movimiento" (citado en Bolos, 2003, 60). Para esto, distingue tres tipos puros de proyectos a partir de los integrantes de los movimientos y de las condiciones estructurales que 


\section{DOXA}

pISSN: 2395-8758

eISSN: en trámite

enfrentan: proyectos religiosos, éticos y políticos.

Estos proyectos se distinguen en que, a) el proyecto religioso "tiene como centro la búsqueda de la regeneración, del cambio en relación a lo trascendente... La especificidad religiosa está en la imputación de esta experiencia y de esta fuerza que se separa y se sitúa en otro lugar desde donde irrumpe en el espacio - tiempo profano" (Bolos, 2003, 61), de manera que, el nuevo Estado requiere una cultura religiosa previa sobre la cual fundamentar la transformación que ha proveído la experiencia de una necesidad vivida más importante y poderosa que el individuo mismo. Mientras que el b) proyecto ético "la experiencia colectiva... encuentra un destino en la liberación, en la transformación, en el renacimiento del individuos y de todos los individuos" modificando el sistema a partir de la transformación de individuos en un proceso gradual, sin confrontaciones directas. Por lo tanto, "exclusividad, secretismo, iniciación y grados de liberación" son características de estos movimientos en algunas de sus etapas (Bolos, 2003, 62).

Por su parte c) en el proyecto político "el grupo es el sujeto de transformación, de reconstrucción de la sociedad. Determina dentro de ella, una "región de socialidad contingente" sobre la que actuará para liberarla siendo el grupo el medio para esa liberación... [Abatiendo] el poder que se opone a esta misión" (Bolos, 2003, 62).

Busca transformar una determinada sociedad y todo está subordinado a este fin, por lo que "sus límites éticos no se encuentran solo en el sistema exterior sino también dentro del grupo mismo. Todo grupo portador de un proyecto político tiene problemas morales propios y, es tanto más político cuanto más los resuelve sobre una base de utilidad política" (Bolos, 2003, 62). Las consideraciones éticas limitarán las acciones del grupo cuando estas no se legitimen por la identidad colectiva y los objetivos del mismo.

\section{El contexto del movimiento por los derechos de género en Baja California}

La decisión de trabajar con el movimiento para la defensa de los derechos de género tiene que ver con el desenvolvimiento de una problemática de violencia hacia la mujer que, sin ser propia o exclusiva de la región, si se presenta como un asunto que toma por sorpresa a la población al 
pISSN: 2395-8758

eISSN: en trámite

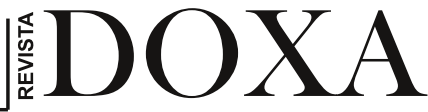

incrementarse el número de incidentes y, por lo tanto, visibilizarse a partir de la cobertura mediática y en las redes sociales.

El presidente de la Corte Interamericana de los Derechos Humanos, Eduardo Ferrer Mc-Gregor Poisot, manifestó que Baja California ocupa uno de los primeros lugares en feminicidios a nivel nacional (Domínguez, 2018). De acuerdo al Libre Movimiento Ciudadano de Baja California en Baja California 6.7 mujeres de cada 10 sufren violencia doméstica: "Tan sólo en 2013 hubo 75 feminicidios, en 2014 registramos 90 y en lo que va del año de 2015 ya tenemos 40 asesinatos de mujeres: 10 asesinadas en Tijuana, 9 en Mexicali, 4 en Tecate. De Rosarito no hay datos. Tijuana ocupa el segundo lugar en feminicidios, después de Acapulco" (Arellano, 2015).

De esta manera, el movimiento por los derechos de género en el estado surge con la consigna de llevar a las calles (y a la vida pública) la visibilización de distintas formas de violencia hacia la mujer y la diversidad sexual en general en la región. Haciendo hincapié en que se trata de un tipo de violencia con características distintas, ya que en ésta persiste la desigualdad, la falta de equidad y la violencia por ser mujeres o por ser "diferentes".

El 27 de enero de 2015 la Red Iberoamericana pro Derechos Humanos presentó una solicitud para emitir una Alerta de Violencia de Género contra las Mujeres (AVGM) en Baja California. De acuerdo a la Comisión Nacional para Prevenir y Erradicar la Violencia contra las Mujeres (CONAVIM), la AVGM es un mecanismo de protección de los derechos humanos de las mujeres único en el mundo. El Artículo 21 de la Ley de Acceso a una vida libre de violencia de 2007, define la Alerta como un conjunto de acciones gubernamentales de emergencia para enfrentar y erradicar la violencia feminicida y/o la existencia de un agravio comparado. $\mathrm{Su}$ objetivo, descrito en el Artículo 23, indica que consiste en garantizar la seguridad de mujeres y niñas, el cese de la violencia en su contra y/o eliminar las desigualdades producidas por una legislación o política pública que agravia sus derechos humanos.

De la misma forma que el movimiento ha avanzado, la normatividad que reconoce y sanciona la violencia contra las mujeres, también lo ha hecho, tanto a nivel federal como a nivel estatal. Una de las más claras manifestaciones es la promulgación en 2007 de la "Ley General de 


\section{DOXA}

pISSN: 2395-8758

eISSN: en trámite

Acceso de las Mujeres a una vida Libre de Violencia”, publicada en el Diario Oficial de la Federación del 1 de febrero de 2007, la cual ha sido reformada en once ocasiones, la más reciente del 13 de abril de $2018 .{ }^{4}$

A pesar de esto, de acuerdo a los activistas estos avances en la Ley aún no se reflejan en la vida cotidiana de las mujeres. En Ensenada, el incremento en feminicidios escala de manera continua desde 2015. De acuerdo a la organización Colectiva de Colectivos Pro Defensa de los Derechos de las Mujeres, en un registro alternativo ${ }^{5}$ que realizan sobre la violencia de género y feminicidos en la localidad, encontraron que en 2016 hubo 11 feminicidios en Ensenada, que casi se duplicó en 2017 a 21 feminicidios (ver Gráfica 1).

Gráfica 1. Feminicidio en Ensenada 2016-2017

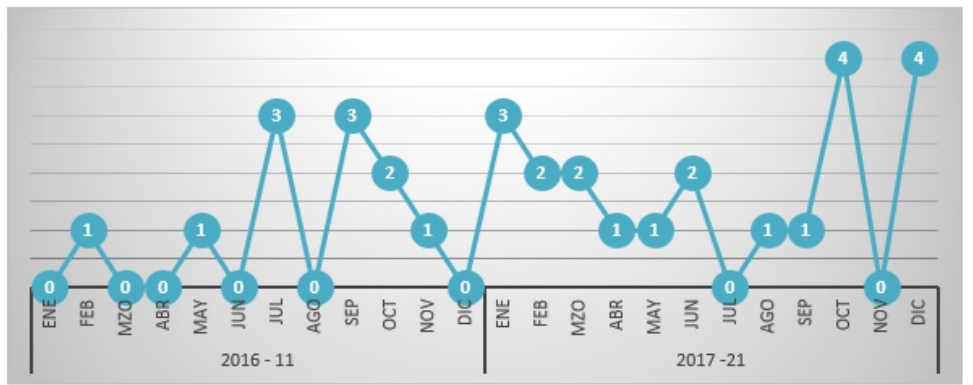

\section{Metodología}

La estrategia metodológica empleada para la investigación en la que se apoya el presente trabajo fue cualitativa; mediante entrevistas y observación

\footnotetext{
3 La Ley General de Acceso de las Mujeres a una Vida Libre de Violencia define diversos tipos de violencia, desde el feminicidio, como el tipo más extremo, hasta la violencia institucional donde "los actos u omisiones de las y los servidores públicos de cualquier orden de gobierno que discriminen o tengan como fin dilatar, obstaculizar o impedir el goce y ejercicio de los derechos humanos de las mujeres así como su acceso al disfrute de políticas públicas destinadas a prevenir, atender, investigar, sancionar y erradicar los diferentes tipos de violencia" (CNDH, 2014, p.6).

4. Los colectivos de la región se han organizado como Colectiva de Colectivos Pro Defensa de los Derechos de las Mujeres y sumado a la campaña de las activistas Claudia Rivera y María Salguero para alimentar el Mapa del feminicidio en México en la plataforma Crowdmap. Esto ante lo que consideran la lentitud del sistema judicial para calificar los asesinatos de mujeres a tiempo, retrasando las posibles acciones que puedan tomarse en las localidades ante el incremento de la violencia de género.
} 
participante se tuvo acceso a los actores principales de este movimiento. La selección de informantes se realizó a partir de su visibilidad, experiencia y conocimiento de expertos sobre el movimiento. Se realizaron entrevistas con 6 líderes que fueron transcritas, categorizadas y codificadas. Apoyamos el proceso análisis con el software Atlas ti.

Para mantener la confidencialidad de la información y anonimato de los activistas, a cada uno se le asignó un código y, con el mismo, se presentan sus testimonios en los resultados.

\section{Resultados}

El incremento y visibilización de fenómenos de acción colectiva que promueven un cambio social con equidad y el fin de la violencia de género, comienza a hacerse ver en Ensenada, en el año 2015, aun cuando acciones similares ya se hacían presentes en los municipios de Tijuana y Mexicali anteriormente.

En sus inicios, se puede observar la presencia de activistas, grupos $\mathrm{y}$ colectivos con distintos grados de formalización y que abarcan un amplio espectro de temas, unidos en torno a la problemática del incremento de la violencia de género en la localidad, principalmente, y el incremento en los feminicidios. Ante esta situación, las manifestaciones del movimiento consistieron en una serie de propuestas de estrategias múltiples y flexibles para presentar las demandas y proponer o construir soluciones.

En el verano de 2015, se realiza por primera vez en Ensenada una marcha que intenta aglutinar las demandas e intereses de los distintos actores sociales bajo una misma bandera, retomando el concepto de La Marcha de las Putas, replicada en diversas localidades de varios países del mundo luego que fuera esta llevada a cabo por primera vez en Toronto, en 2011. En Ensenada, el 27 junio 2015, más de 100 mujeres, varones, niñas y niños marcharon, con el propósito de "concientizar sobre los niveles de violencia que viven las mujeres en nuestro día a día, desde el acoso callejero hasta el feminicidio, y hacer visible la estructura patriarcal que tanto hombres como mujeres ha inculcado y que hoy no es compatible con todos los derechos humanos universales".

A partir de este evento, se logran identificar la diversidad de los integrantes del movimiento, así como, por las afinidades del trabajo 


\section{DOXA}

pISSN: $2395-8758$

eISSN: en trámite

colectivo, se forman nuevas alianzas entre ellos. De las mismas, son visibles a la fecha organizaciones, grupos y colectivos como: Comunidad LGTB de Ensenada, el Consejo para la protección de los derechos de la diversidad sexual (CPDDS Ensenada), Diversidad Sexual de Ensenada, A.C., Colectivo DIVERSA, grupo de Mujeres bordadoras contra el olvido, Colectivo \#26N, Movimiento \#24A, Colectiva de Colectivos Pro Defensa de los Derechos de las Mujeres, entre otros.

\section{La organización del movimiento y sus estrategias}

Ensenada es una ciudad portuaria del noroeste de México que, actualmente, es considerada con buena calidad de vida. Sin embargo, también ha sido calificada por activistas sociales como una localidad con poca participación, algo elitista y conservadora; con poca respuesta organizativa a demandas públicas que tienen respuestas masivas en otros lugares del país.

Ser un movimiento social en Ensenada, de acuerdo a los activistas del movimiento por los derechos de género, tiene limitaciones:

"Ensenada siempre es lenta para reaccionar, Ensenada reacciona muy lento a diferencia del centro del país... Siempre he dicho que la falta de que, de que en Ensenada realmente todos somos de fuera y no tenemos arraigo con la tierra, no tenemos arraigo con las familias, no tenemos arraigo histórico y entonces esto hace que las, que las cosas funcionen con menos amor por la patria, con menos amor por el terruño y por eso somos creo tan indiferentes a los grandes problemas" (A2).

Para el caso de este movimiento, particularidades de Ensenada como la existencia de grupos conservadores y religiosos con influencia en la comunidad, han contribuido a que sus demandas no sean entendidas o bien recibidas en todos los sectores sociales:

"Hubo personas que cuestionaron, que atacaron, personas que quisieron que no se hiciera la marcha y fue un proceso de autoexploración porque sabíamos que estábamos muy comprometidos con la causa, sabíamos que se tenía que hacer algo en Ensenada, que era algo histórico, por las dimensiones que tiene y sabíamos que iba a raspar muchas fibras sensibles morales en una ciudad como Ensenada y fue un proceso, si, desgastante" (A8).

El movimiento por los derechos de género en Ensenada se 
caracteriza por ser un colectivo, asociativo. Sin embargo, no se puede considerar homogéneo, ya que aun cuando han logrado articular sus acciones y manifestarse como un frente unido en algunos momentos, la diversidad de los actores que lo componen ha contribuido a momentos de tensión dentro del mismo. Una de las características que presenta es la diversidad en las formas que toma el activismo de los participantes.

La forma, los discursos y las estrategias que adquiere el movimiento se negocian desde el interior del mismo, creándose un campo de reflexión y conflicto al encontrarse posiciones distintas. Estos cuestionamientos se contraponen con los de las activistas más convencidas de que, una vez que adquieres conciencia del problema, es un deber salir a la calle y compartirlo:

“¿Si fuese un movimiento manipulatoro? y si ¿sí lo fuese?, ¿si fuese un movimiento manipulatorio que está visualizando la violencia machista, que está visualizando la falta de perspectiva de género, que está visualizando la naturalización y la normalización de la violencia en nuestro país?, si alguien manipuló todo eso es malo. [Pero es más] malo que nos solidaricemos a no normalizar la violencia, es malo que nos manipulen, ...es malo que alguien nos esté manipulando para que no estemos en contra de quienes gobiernan sin perspectiva de género ¿cómo? o sea, mejor cállate la boca, porque si trascendió ¿no? si hubo quien se solidarizó" (A2).

De acuerdo a la activista $\mathrm{A} 5$, un riesgo que corren los movimientos como éste es el realizar activismo para los activistas:

"no un activismo para la clase que se necesita formar y esa es una de mis preguntas ¿cómo convocas a la ciudadanía, mujer, independientemente que sepa de género?... Si yo le digo a una chica "Oye, fíjate que hay alerta de género", [Me va a responder] ¿Qué es eso? Sí, es como un término académico para un asunto social... Entonces, pues, si a mí me queda claro yo estudié eso, pero tendría que quedarle claro a las mujeres de 14, 17, 18 años qué significa o qué está pasando en la ciudad."

Los discursos, las posiciones ideológicas sobre el tema están construidas con referentes que no tienen la mayor parte de la población, por lo tanto el éxito del movimiento se verá disminuido al no encontrar eco en los sectores sociales. 
Un problema que enfrentan los activistas al interior del movimiento es el ego:

"si yo he trabajado con género pero si no se me llamó desde el inicio pues no me sumo, entonces en lugar de eso resto, ...eso también es algo que tenemos que trabajar todos los activistas: que la mayoría quiere iniciar un proyecto o estar al frente de un proyecto y cuando no es así a veces no se suman, yo conozco varias feministas y varios hombres en pro del feminismo que no estaban ahí y que tenía mucho que ver con este problema de los egos" (A8).

Por otra parte, hay visiones más conciliadoras:

"También ellas manejan unos rollos bien heteropatriarcales y de las cosas tienen que ser así, yo con eso me enfrenté, y dije nooo... Creo que a muchas nos falta algo de auto observación y de respeto, también, a las ideas y a las formas diferentes de ver los mismos temas" (A7).

Comentan los activistas que es necesario reflexionar sobre la apertura del movimiento y la membresía al mismo, ya que el celo sobre el tema y las reivindicaciones que lleva consigo hace, en ocasiones, que se cierren las puertas a personas valiosas porque no tienen "el curriculum" necesario o porque son hombres.

"Cuando yo empecé a involucrarme con esto, si había como ese recelo... como cuando entras a un trabajo son 3 meses de prueba para ver si te quedas no. Cuando a mí me invitaban si asistía, pero si veía los cuestionamientos o esa falta de inclusión inmediata, paradójicamente, ¿no?, esa falta e inclusión o invitación. Hasta que sobre la marcha me fui dando a conocer y cuál era mi perfil o mi interés en todo esto y ya ahorita te digo que tengo muy buena relación con todas las feministas de aquí, pero si generaba mucho eso, ver a un hombre que genuinamente se incluya en la causa feminista. ...Creo que mi inclusión representa el romper con el estigma de que el feminismo es la lucha en contra del hombre" (A8).

\section{Liderazgo y motivaciones para la acción}

La participación de cada integrante del movimiento es muy similar a otros fenómenos de acción colectiva, surge por inquietudes y experiencias personales o cercanas que los estimularon a involucrarse para la transformación de las condiciones observadas, en este caso, la inequidad y la 
pISSN: 2395-8758

eISSN: en trámite

violencia de género.

En la trayectoria de los activistas involucrados se observa que no es su primera experiencia en este tipo de acciones, donde algunos vivieron experiencias desde la casa familiar, lo que ha construido una vocación a la participación en beneficio de grupos vulnerables. Expresa A7, quien desde muy joven escuchó a su padre hablar de la importancia de participar en colectividad y más aún, en entornos de extrema desigualdad, como son las zonas rurales del país: "me siento con la responsabilidad social si no de intervenir, por lo menos de monitorear".

En la búsqueda por contribuir a la solución de las problemáticas observadas, los activistas han coincidido en diversos espacios, han establecido vínculos y buscado construir una agenda conjunta para visibilizar y desnaturalizar la violencia de género, la falta de equidad y la importancia de reconocer y respetar la diversidad sexual; en el camino han propiciado la conversación sobre estos temas en distintas esferas y sumado la participación de más actores.

Si bien algunos de los integrantes del movimiento han desarrollado parte importante de su labor en contextos rurales, están conscientes de que la violencia de género afecta a mujeres de todos los estratos sociales.

"se piensa muchas veces que la violencia de género se da en las colonias de las orillas o en casos de personas con un nivel socioeconómico bajo, pero no es cierto, se da en todos los niveles, personas profesionistas, personas preparadas, el sistema patriarcal no respeta..." (A8).

Como parte de su involucramiento en la esfera pública, los integrantes del movimiento han constatado la diversidad de formas en que la violencia de género se expresa y cómo permea el ejercicio de lo político, de tal forma en que se han enfrentado a la dificultad de ser escuchados y de que sus propuestas sean tomadas en cuenta

“está tan naturalizada la violencia política hacia la mujer que no se dan cuenta cuando cierran un círculo y te dejan fuera de él, no se dan cuenta cuando te interrumpen, no se dan cuenta cuando no te escuchan" (A7).

Por otra parte, han sido testigos de cómo la violencia institucional suele "revictimizar" a quienes enfrentan condiciones de violencia, al no comprender la complejidad de los problemas que enfrentan.

"yo creo que hay personas con buenas intenciones pero hace falta mucha 
profesionalización y una conciencia más amplia que tenga que ver con problemas de base y no solamente asuntos mediáticos" expresó A8.

Como parte de su trayectoria, los activistas han atestiguado la naturalización y justificación de la violencia, incluso en mujeres funcionarias:

"Te encuentras en las mujeres gobernantes un profundo machismo y una profunda violencia machista en sus ejercicios y en sus funciones como funcionarias o como gobernantes... al ser líderes femeninas pues muchas otras mujeres van a seguir sus movimientos, y van a reproducir en la sociedad exactamente las formas que están viendo en sus líderes... y entonces esa va a hacer la parte más difícil de combatir en nuestra sociedad, el machismo en la mujer, no?" (A2).

Los activistas subrayan la necesidad de transformar y transparentar el ejercicio gubernamental, ya que "el tema que ha lastimado nuestra calidad de vida es la corrupción, la impunidad y la ineficiencia de los servidores públicos" ( ); por otra parte "la política ya no va a poder ser a las altas horas de la noche o en un table dance... se va a dignificar" (A7).

La experiencia de haber vivido de manera cercana diferentes tipos de violencia y discriminación ha creado un mayor compromiso de sus integrantes con las exigencias del movimiento, tal como como lo relata una de sus miembros:

"Todo el tiempo somos víctimas todo el abanico de violencia... somos víctimas del transfeminismo...de la discriminación...de las leyes... de las instituciones y entonces esto hace que...el colectivo trans tenga una gran cantidad de participantes activistas en el feminismo... y que participan de fondo, es decir, no participan solamente llevando la pancarta no, participan con opinión en los movimientos, en el corazón de los movimientos, en la logística y en la inteligencia de los movimientos" (A2).

\section{Dimensión politica del movimiento}

El movimiento por los derechos de género en Ensenada procede como una organización informal; lo integran diversas organizaciones y colectivos, que se han incorporado al movimiento como resultado de las interacciones que tienen lugar a partir de intereses comunes y de coincidencias en el espacio urbano, como en los cafés y bares, por ejemplo, pero también a través del espacio virtual: "las redes sociales nos acercan a las 
personas que simpatizan y nos ayudan a generar la reflexión de quienes no simpatizan para de ahí hacer contacto directo... lo hemos hecho y ha funcionado." (A8). Para convocatorias como para visibilizar problemas las redes sociales son muy útiles.

El movimiento crece a partir de alianzas con grupos con problemáticas similares. A4 comenta que es en las actividades o pláticas que los invitan a dar, que se les acercan personas motivadas, que los quieren ayudar. Así han formado sus redes con asociaciones como HF México y otras en San Diego para cuestiones de VIH. Lo mismo que las problemáticas, las alianzas y apoyos también trascienden las fronteras, la misma marcha tiene manifestaciones nacionales e internacionales.

Los colectivos y organizaciones participantes tienen en común la lucha por el reconocimiento de los derechos de grupos tradicionalmente vulnerados y la búsqueda de la sensibilización de la comunidad. Si bien las experiencias vividas pueden haber sido diferentes, parten de una falta de reconocimiento a sus derechos y eso los coloca en situaciones similares para el análisis de su realidad. El movimiento por los derechos en Ensenada y sus manifestaciones.

"Es un colectivo... y el colectivo marcha de las putas puede ser cualquiera, y eso es lo importante: que el mensaje se siga dando y que sigan existiendo voces y que las mujeres se sigan empoderando" (A7).

En el movimiento se distingue el proyecto político, transformador, como la motivación para la acción de los activistas que participan en el mismo:

"Bueno, pues hay que empezar a trabajar un poquito en eso, vamos a tratar de hacer un cambio y prácticamente desde que nació comunidad LGTB de Ensenada trabaja por medio de Facebook" (A4).

Esta idea transformadora se extiende a todos los sectores sociales, incluso a los mismos actores que componen o se identifican con el movimiento, ya que:

"dDntro de la misma comunidad hay discriminación y hay mensajes de odio entre gays, lesbianas, transgénero, transexuales... porque el sistema patriarcal nos enseña que lo masculino siempre va a tener más poder, siempre va a ser más fuerte. Entonces, va más allá de respetar a la comunidad LGBTI si no va a romper de los esquemas de pensamiento que 
están dentro de la comunidad y fuera de la comunidad" (A8).

Para lograr su propósito transformador resulta indispensable la conjunción de esfuerzos de colectivos y de organizaciones con objetivos similares. Pero también las simpatías individuales pueden contribuir con este propósito:

"La forma como nos acercábamos inicialmente [a las dependencias públicas o escuelas] fue institucional, pero no había una respuesta institucional, como de yo no te lo puedo autorizar... o yo no te puedo decir que autorizo por escrito que promociones aquí la marcha de las putas, pero pasa y pega los carteles" (A8).

Asimismo, este movimiento encontró en el sistema político tuvieron antagonistas importantes, que debían cuidar para mantener el objetivo del movimiento:

"Cuando llevas rato en los movimientos y en las movilizaciones un tema que te preocupa es el tema de los infiltrados entonces siempre te preocupa el que pudiese haber manipulación, es decir el que alguien pudiese haber sido quien prendió el movimiento, quien vitalizo el movimiento con fines manipulatorios. Esto lo sabes, cuando vienes del activismo siempre debes tener mucho cuidado con estos temas y te tienes que salir y tienes que decir haber es auténtico o no es auténtico, es decir, ¿Alguien le prendió fuego a esto?" (A2).

Considerando la diversidad de los activistas, es de esperarse que si bien hay acuerdo en lo general, cuando se trata de particularidades, haya diferencias. Lo anterior es válido en lo que se refiere a la relación y/o afiliación partidista. Mientras que A8 prefiere mantenerse al margen de los partidos, A7 fue candidata a diputada local por un partido político. "Es bien difícil mantener como tu asociación, tu persona y luego todavía tu ideología con un partido político" (A4).

Lo anterior puede representar un riesgo para el movimiento, ya que las consideraciones éticas limitarán las acciones del grupo cuando estas no se legitimen por la identidad colectiva y los objetivos del mismo (Bolos, 2003).

\section{Alcances del movimiento}

Los alcances del movimiento están relacionados con la visión colectiva hacia el interior y de sí mismos de los actores sociales, del 
pISSN: 2395-8758

eISSN: en trámite

cuestionamiento que hacen a sus estrategias y el manejo a las críticas internas y externas:

"ahorita la verdad es que yo me cuestiono mucho que tanto logramos a través de del activismo, no en cuanto a la información que permea en la gente, sino en las políticas públicas... yo ahí me cuestiono un poco, ¿no? Entonces, creo que la gente dice: al cabo no vamos a lograr nada, para qué vamos, para qué marchamos, es más lo que nos arriesgamos porque pueden haber infiltrados, pueden haber pleitos pueden haber trancazos, y la gente desiste de ir" (A7).

Sin embargo, la suma (o resta) de las acciones por la visibilidad y concientización sobre el problema y la declinación de participación, en algunos casos, es positiva hasta el momento. Esto se puede observar en la creciente presencia del tema en diversos espacios, principalmente en las redes sociales locales y regionales, donde se debaten temas coyunturales con participación de distintos puntos de vista.

$\mathrm{Al}$ respecto, otro aspecto a resaltar es el número de "asociaciones spin-off" que surgieron del movimiento inicial de 2015. Estas se presentan más especializadas en las temáticas que tratan, con demandas más concretas y para grupos de población específicos, como las mujeres y niñas, la comunidad LGBT, las mujeres trans, entre otros. Estas organizaciones han seguido las estrategias de mantenerse vivas en redes sociales y realizar actividades directas en la comunidad, lo que las ha mantenido con vigencia y han incrementado sus redes de apoyo.

Esta capacidad de diversificación de la agenda, apoyando ejercicios similares de acción colectiva de colectivos nuevos y con la creación de redes presenta en la actualidad un panorama esperanzador para el movimiento de los derechos de género en Ensenada. Se presenta ahora la necesidad de construir estrategias que permitan a los grupos fortalecerse para tener mayor penetración en los distintos estratos sociales, de manera que esto favorezca llevar a la agenda pública sus demandas.

\section{Conclusiones}

El estudio de los nuevos movimientos sociales en localidades medias aporta, tanto a la academia como a los activistas mismos, herramientas para comprender los fenómenos de acción colectiva en diferentes niveles. Desde 


\section{DOXA}

pISSN: 2395-8758

eISSN: en trámite

esta perspectiva, y principalmente retomando la postura de los teóricos de la identidad, fue posible analizar los procesos al interior y exterior del fenómeno en Ensenada, ubicando el proceso de construcción de una identidad colectiva a partir de las individualidades de sus integrantes, los recursos con que cuentan (materiales e ideológicos), sus apoyos y antagonistas. Para la comprensión de este caso, ha probado la vigencia de sus postulados, dado que las demandas que llevaron a su surgimiento siguen vigentes, como en el caso de la defensa de los derechos de género.

Los activismos (femeninos y masculinos) en las demandas de género en Ensenada, se relacionan con la percepción del enquistamiento del sistema heteropatriarcal en la sociedad y observamos que la flexibilidad de las estrategias de acción que las características mismas de los movimientos sociales fomentaban, se fortalecen y retroalimentan en el mundo virtual. Así mismo, vemos como este movimiento se ha capitalizado creando "acontecimientos mediáticos" (Kaldor, 2005) e incorporando en sus acciones la cobertura a través de redes sociales como la estrategia principal para generar respuesta social.

Las novedades de estos nuevos movimientos sociales se presentan en su composición y en la transformación de su autopercepción, más libre, flexible y organizada ahora en "colectivos" y no en asociaciones u ONGs, como en el pasado. Con un arsenal de herramientas para la visibilización de demandas y denunciar la que polarización de los derechos la dicta el heteropatriarcado, toman la calle y a través de la marcha, el performance y las redes, se la apropian.

\section{Referencias}

Aguirre, R. (2003). Género, ciudadanía social y trabajo. Montevideo: Doble Clic Editoras.

Arellano García, C. (2015, marzo 25). Se incrementan feminicidios en Baja California y Guanajuato. La Jornada de Baja California, consultado el 30 de julio de 2018 en: http://jornadabc.mx/tijuana/25-032015/se-incrementan-feminicidios-en-baja-california-y-guanajuato.

Becerra Pozos, L. \& Mata, E. (2014). Organizadas es mejor. Manual para la incidencia en política pública desde la perspectiva de la mujer indígena. Oaxaca: Iniciativas para el Desarrollo de la Mujer 
pISSN: 2395-8758

eISSN: en trámite

Oaxaqueña, A.C. (IDEMO, A.C.).

Bolos, S. (2003). Organizaciones sociales y gobiernos municipales. México: Universidad Iberoamericana.

Comisión Nacional de los Derechos Humanos (2014). Violencia institucional contra las mujeres, México: CNDH.

Dalton, R. J. (2008), Citizenship norms and the expansion of political participation. Political Studies, 56: 76-98.

De Keijzer. (1998). El varón como factor de riesgo. Villahermosa: ECOSURUJAD.

Delhumeau Rivera, S. (2012). Organizaciones no gubernamentales en Tijuana y el gobierno local de la alternancia (1988-2000). Mexicali, Baja California: Universidad Autónoma de Baja California.

Domínguez, A. (2018). BC de los Estados con más feminicidios. La Voz de la Frontera. Recuperado el Recuperado el 29 de agosto 2018 de: https://www.lavozdelafrontera.com.mx/local/bc-de-los-estadoscon-mas-feminicidios-1038284.html

Gallo, L., Correa Mejía, J.A. \& Pacheco Danies, A.M. (1997). Procesos de desarrollo comunitario asociados a los diferentes estilos de liderazgo en las comunidades negras de la ciudad de Barranquilla. Investigación y Desarrollo, (6), 97-104.

Hernández García, Y. (2006). Acerca del género como categoría analítica. Nómadas. Critical Journal of Social and Juridical Sciences, 13(1), $111-120$.

Kaldor, Mary (2005). La sociedad civil global. Una respuesta a la guerra. España: Tusquet Editores.

Lamas, M. (2004). Qué generó el género. En Mujeres al timón de la función pública. Instituto de Liderazgo Simone de Beauvoir A.C., México.

López Estrada, S. (2002). Las organizaciones no gubernamentales feministas en Baja California. En Baja California: escenarios para el nuevo milenio (203-230). Distrito Federal: Universidad Nacional Autónoma de México.

McCarthy, J.D. \& Zald, M.N. (1977). Resource mobilization and social movements: A partial theory, American Journal of Sociology, 82(6), 1212-1241. 
Melucci, A. (1985). The symbolic challenge of contemporary social movements. Social change, 52(4), 789-816.

Melucci, A. (1988). Nomads of the present. Social movements and individual needs in contemporary society. Philadelphia: The Temple University Press.

Molina Luque, F., Samper Rasero, L., \& Mayoral Arque, D. (2013). Liderazgo femenino. Un análisis de las diferencias de género en la formación y desarrollo de asociaciones de inmigrantes africanos. Revista Internacional de Sociología, 71 (Extra_1), 141-166.

Moreno Mena, J.A. (2008). Tejedores de esperanza: redes de organizaciones civiles en Baja California. Mexicali, Baja California: Universidad Autónoma de Baja California.

Offe, C. (1985). New social movements: challenging the boundaries of institutional politics. Social research, 52(4), 817-868.

Oraisón, M. (2011). Sobre la relación Estado - Sociedad civil: la participación ciudadana. El caso de las comisiones vecinales de la ciudad de Corrientes, Argentina. Nómadas, Revista crítica de Ciencias Sociales y Jurídicas, Número especial, 289-306.

Pedrín Rembao, K. J., Delhumeau Rivera, S., Spears Kirkland, A. y Martínez Valdés, G. C. (2014). ¿Por qué me callo, por qué me dejo?: el empoderamiento de Lila. En Mujeres en espacios cambiantes: familia, trabajo y colectividades (161-84). Mexicali: Universidad Autónoma de Baja California.

Reyes Ruiz, M. (2006). Organizaciones no gubernamentales en Baja California: el caso de los grupos ambientalistas y de mujeres. Mexicali, Baja California: Universidad Autónoma de Baja California.

Rojas Andrade, R. (2013). El liderazgo comunitario y su importancia en la intervención comunitaria. Psicología para América Latina, (25), 5776.

Silva, C. \& Loreto Martínez, M. (2004). Empoderamiento: proceso, nivel y contexto. Psykhe, 13 (2), 29-39

Tarrow, S. (1998). Power in movement. 2d ed., Cambridge: Cambridge University Press.

Tilly, C. (1995). Las revoluciones europeas, 1492-1992. Barcelona: Crítica. 
pISSN: 2395-8758

eISSN: en trámite

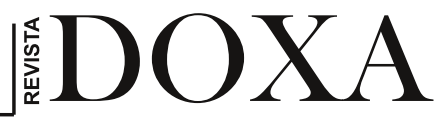

Touraine, A. (1981). The voice and the eye: an analysis of social movements. Cambridge: Cambridge University Press. 